A novel all-fibre configuration for a flexible polarimetric current sensor

This article has been downloaded from IOPscience. Please scroll down to see the full text article.

2004 Meas. Sci. Technol. 151525

(http://iopscience.iop.org/0957-0233/15/8/016)

View the table of contents for this issue, or go to the journal homepage for more

Download details:

IP Address: 128.178.81.84

The article was downloaded on 01/02/2011 at 17:00

Please note that terms and conditions apply. 


\title{
A novel all-fibre configuration for a flexible polarimetric current sensor
}

\author{
Dario Alasia and Luc Thévenaz \\ EPFL, Swiss Federal Institute of Technology, Nanophotonics and Metrology Lab, \\ CH-1015 Lausanne, Switzerland \\ E-mail: dario.alasia@epfl.ch and luc.thevenaz@epfl.ch
}

Received 19 January 2004, in final form 11 May 2004

Published 19 July 2004

Online at stacks.iop.org/MST/15/1525

doi:10.1088/0957-0233/15/8/016

\begin{abstract}
In this paper a polarimetric fibre-optics current sensor in a new highly versatile all-fibre configuration is described. The optical configuration is particularly simple and is based on a back-and-forth propagation through a twisted sensing fibre. This results in a very flexible sensing cable which can be adapted to a great number of existing power installations, by simply winding the fibre around the uninterrupted electrical conductor. The prototype presented here is designed to measure ac and dc currents with an excellent accuracy over more than three decades.
\end{abstract}

Keywords: optical fibre, current sensor, polarimetric sensor, Faraday effect

(Some figures in this article are in colour only in the electronic version)

\section{Introduction}

Fibre-optics current sensors offer many advantages compared to classical current transformers. The small sizes of optical fibres facilitate the installation of this kind of sensor and the intrinsic insulation due to their dielectric nature represents a significant improvement for high-voltage installations. Furthermore, they show a high bandwidth making the observation of harmonics and transients possible and offering a large immunity to stray magnetic fields. Finally, their sensitivity and the absence of saturation of the magneto-optic effect make it possible to measure high currents (up to $500 \mathrm{kA}$ ) [1] as easily as low currents (some amperes).

Producing commercial optical current sensors is still challenging and prototypes are developed basing on three different schemes: bulk-optics, fibre-interferometric and fibrepolarimetric sensors. Bulk-current sensors [2] are made using high Verdet constant crystals, resulting in excellent sensitivity, but are subject to alignment and temperature drifts. Optical fibres show a lower Verdet constant, but increasing the number of turns of fibre wound around the conductor results in an improved sensitivity. The interferometric configuration, using a Sagnac interferometer [3], measures the non-reciprocal phase shift with a high accuracy, but the main reported problem is the high vibration sensitivity. The polarimetric method simply measures the rotation of a linear polarization [4] but such sensors require a precise orientation of the analyser.

As a general feature, sensors based on single-mode fibres are sensitive to the variations of the sensing-fibre birefringence which is responsible for changing the polarization along the fibre. Many solutions have been proposed to compensate the effect of birefringence, either optically or through a proper signal processing $[3,5]$. But these techniques are limited to homogeneous linear birefringence along the sensing fibre, so that they turn out to be widely inapplicable in actual conditions in which the fibre birefringence is basically random.

Fortunately the detrimental effect of polarization mode dispersion in telecommunication systems has led to a big effort for manufacturing very low birefringence fibres at low cost. The remaining birefringence of such fibres turns out to still be too large for the proper operation of a current sensor, but it can now be widely rendered negligible by annealing [6] or mechanically twisting [7], so that a circular or freely-rotating linear polarization is maintained over the entire fibre length.

An original polarimetric configuration using a free-space in-line Faraday rotator is reported in [8]. This sensor has the key advantage to require no adjustment of any kind to the optical elements and its accuracy is poorly dependent on the optical elements tolerance (an extensive study may be found in [9]). However, this advantage vanishes as soon as any polarization-transforming element is inserted after the 


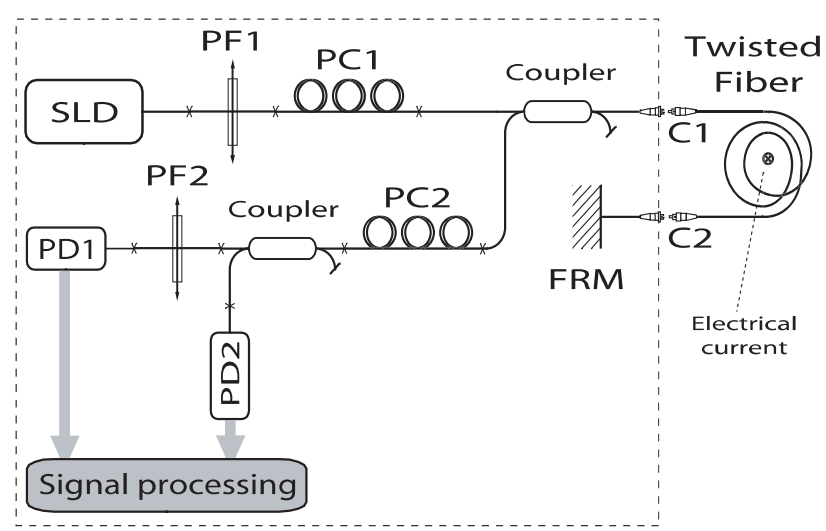

Figure 1. Schematic diagram of the all-fibre polarimetric current sensor. Fibre polarizer PF1 polarizes light generated from a super luminescent diode (SLD). Polarization controller PC1 is set to make the polarization in the sensing head linear. The twisted fibre maintains the polarization up to the Faraday mirror. Reflected light is coupled towards PC2 which is set to yield a linear polarization rotated by $45^{\circ}$ at the entry of the fibre polarizer PF2. The outgoing light is collected by photodetector PD1. PD2 measures the total intensity of light $\left(I_{0}\right)$.

Faraday rotator, in particular jumpers and connectors. These practical problems occur when leaving the laboratory bench to contemplate an industrial prototype of the sensor in which a connecting fibre is needed to link the sensing head to the instrument. Since such a connecting fibre shows random birefringence, the linear polarization at the rotator output is not preserved and a polarization controller must be inserted to compensate the linear birefringence. But, as the main asset of this sensor consists in the total absence of adjustment the concept of an in-line rotator no longer makes sense and the configuration has been entirely revisited to result in a novel all-fibre polarimetric configuration, which is presented in this paper.

\section{Sensor description}

The optical configuration is quite simple and based on backand-forth propagation through the sensing head, as shown in figure 1. The light generated from a super luminescent diode (SLD) is linearly polarized by travelling through a polarizing fibre. Assuming that the electrical current is zero, the polarization controller PC1 is adjusted in order to compensate the linear birefringence due to connectors and to have a linear polarization right after the input connector $\mathrm{C} 1$ of the sensing head. The sensing fibre is mechanically twisted and is thus predominantly circularly birefringent [7]; in this way, light is kept linearly polarized while propagating through it. The light is then reflected back by the Faraday rotation mirror (FRM) and the result is again linearly polarized right after the output connector $\mathrm{C} 2$, though orthogonally to the incident wave. This way the polarization is guaranteed to be linear during the back propagation through the twisted sensing fibre, the reciprocal rotation due to twisting is automatically cancelled out and any small residual linear birefringence is compensated.

Further, on the way back, the coupler redirects light towards the second polarization controller PC2, which has the function to set the polarization at $45^{\circ}$ with respect to the polarizer axis (PF2).

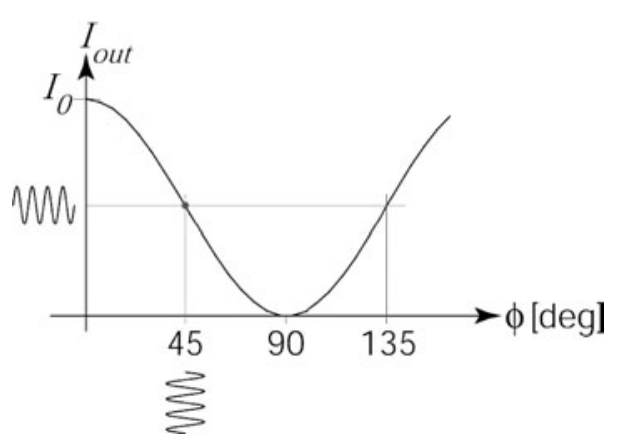

Figure 2. Polarizer response: transmitted light intensity versus polarizer orientation. The $45^{\circ}$ operating point results in a linear variation of the transmitted intensity for any small rotation of the input polarization.

The linear polarization is therefore just set at the halftransmitting point of the polarizer, so that any small rotation of the polarization-like that caused by an electrical currentresults in a linear variation of the transmitted intensity, as depicted in figure 2 .

When an ac current circulates in a conductor enclosed within the sensing fibre, the linearly polarized light experiences a rotation due to the non-reciprocal Faraday effect. Adopting the signal processing described in [8], the electrical current is directly calculated from ac and dc components of the output intensity:

$$
\begin{aligned}
& I_{\mathrm{el}}^{\mathrm{ac}}(t)=-\frac{1}{4 N V} \arcsin \left(\frac{I_{\mathrm{ac}}(t)}{I_{\mathrm{dc}}}\right) \approx-\frac{1}{4 N V} \frac{I_{\mathrm{ac}}(t)}{I_{\mathrm{dc}}} \\
& \text { for } I_{\mathrm{ac}}(t) \ll I_{\mathrm{dc}}
\end{aligned}
$$

$N$ being the number of turns of fibre enclosing the conductor and $V$ the Verdet constant.

If the operating point is slightly shifted off the centre of the linear polarizer response, the current response becomes partially nonlinear and can be conveniently approximated by a third-order polynomial, with no actual penalty.

\section{Sensing head flexibility}

To make the system even more immune to a possible residual birefringence in the sensing head, a Faraday rotation mirror is used instead of a standard mirror. The sensing fibre is never strictly free of linear birefringence-even after significant twisting - so that the use of a FRM cancels to a wide extent the effect of any residual birefringence and its variations. In the absence of an electrical current the polarization of the backward propagating light is, at any point, orthogonal to the polarization of the light travelling in the forward direction, making the effect of any linear birefringence cancelled [10]. This particular case can be generalized to any state of polarization by the usual expansion on the eigenaxes of the fibre birefringence.

In this way, it is possible to have a very flexible sensing cable which is adaptable to a great number of different installations and sensing requirements, and moreover is vibration insensitive. Figure 3 shows a measure of the polarization state of the backward-propagating light when mechanical vibrations are present. It is possible to note that the point representing a polarization state on the Poincaré sphere 


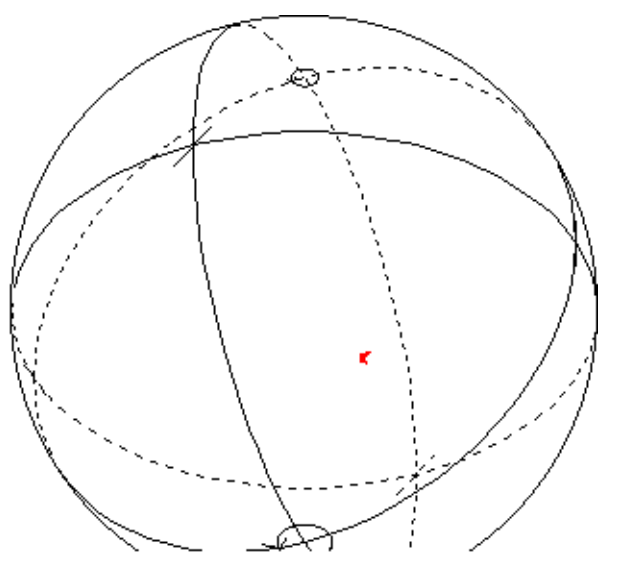

Figure 3. Measurement of the polarization state stability of the backward propagating light when mechanical vibrations are present. The point representing the polarization state on the Poincare sphere is stationary while the cable is subject to a significant change of its geometry (from a circle to a figure- 8 shape).

is stationary while the sensing cable is subject to a significant change of its geometry, for instance from a circle to a figure- 8 shape.

\section{Adjustment of the polarization controllers}

\subsection{Adjustment of controller PCI}

The correct adjustment of the polarization controllers represents the most delicate procedure required by this experimental configuration. The first polarization controller (PC1) is set by observing the polarization state of the forward propagating light at the output of the sensing fibre on a polarization analyser. As a result of the linear birefringence due to connectors the state described by a point on the Poincare sphere does not inform on the actual state of polarization along the twisted fibre. We thus need to operate an indirect measure in order to establish whether the propagating polarization state is linear or not.

A simple technique consists in significantly changing the optical path length of the sensing fibre by actively modifying its geometry. Since the eigenstates associated with a twisted fibre are left- or right-circular polarization states, a variation of the birefringence delay does not affect the propagation of circularly polarized light. This means that a point representing a circular polarization state will be stationary on the Poincaré sphere, while moving the sensing cable.

In contrast, as a linear polarization state is very sensitive even to a minor change of the birefringence delay, it will draw a full circle on the Poincaré sphere while moving the sensing cable. The polarization is thus adjusted by measuring its state at the output of the fibre in order to match the situation shown in figure 4.

\subsection{Adjustment of controller PC2}

An efficient method to properly set the second polarization controller (PC2) consists in maximizing the ac component of the output light intensity with a nonzero reference electrical current enclosed within the sensing head.

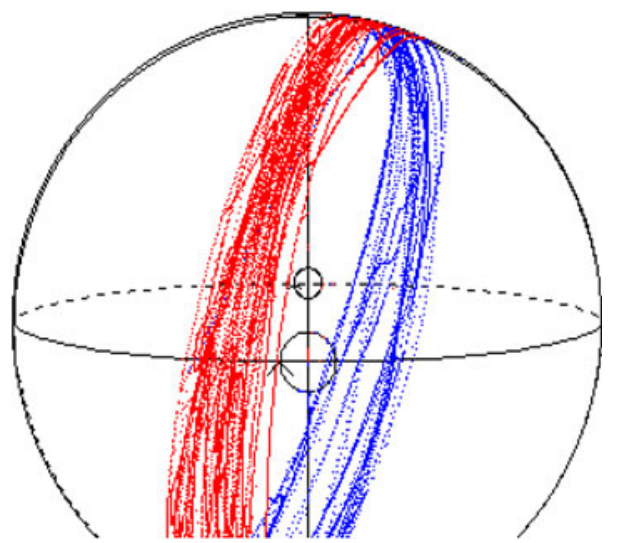

Figure 4. Measurement of the polarization state of the forward propagating light at the output of the sensing fibre. A point representing a linear polarization state draws a full circle on the Poincaré sphere while moving the sensing cable.

The polarizer response (PF2) to a general (elliptical) polarization state is given by

$$
I=I_{0}\left(\cos ^{2} \phi \cos ^{2} \varepsilon+\sin ^{2} \phi \sin ^{2} \varepsilon\right)
$$

where $\phi$ and $\varepsilon$ represent respectively the polarization orientation and the ellipticity angle. The sensitivity to small variations of orientation is simply the derivative with respect to $\phi$ :

$$
\frac{\mathrm{d} I}{\mathrm{~d} \phi}=-I_{0} \sin 2 \phi \cos 2 \varepsilon
$$

It is evident that the ac output signal will be maximized only if the sensitivity (3) is maximized:

$$
\max \frac{\mathrm{d} I}{\mathrm{~d} \phi} \longleftrightarrow \phi=\frac{\pi}{4}, \quad \varepsilon=0 .
$$

Maximizing the ac component results in having a linear polarization and in setting the operating point exactly at $45^{\circ}$.

\section{Modelling}

A correct adjustment of the polarization controllers may result in a quite delicate and critical operation (especially for controller PC2). For this reason, the development of proper signal processing, capable of taking into account the nonidealities present in the system, would be a clear advantage.

We suppose now that the lightwave is not perfectly linearly polarized $(\varepsilon \neq 0)$ and that the operating point is not exactly set $\left(\phi_{0} \neq 45^{\circ}\right)$. Since the Faraday rotation due to an electrical current is given by $\phi_{\mathrm{F}}(t)=N V I_{\mathrm{el}}(t)$, equation (2) is thus modified:

$$
I=\frac{I_{0}}{2}\left[1+\cos 2 \varepsilon \cos \left(2 \phi_{0}+4 \phi_{\mathrm{F}}(t)\right)\right]
$$

The ac and dc components of the output intensity may be calculated analytically using the Jacobi-Anger expansions of trigonometric functions into series of Bessel functions:

$$
I_{\mathrm{dc}}=\frac{I_{0}}{2}\left[1+\cos 2 \varepsilon \cos 2 \varphi_{0} J_{0}\left(4 \hat{\phi}_{\mathrm{F}}\right)\right]
$$

$I_{\mathrm{ac}}^{\mathrm{rms}}=\cos 2 \varepsilon \frac{I_{0}}{2} \sqrt{\frac{1}{2}+\frac{1}{2} \cos 4 \varphi_{0} J_{0}\left(8 \hat{\phi}_{\mathrm{F}}\right)-\cos ^{2} 2 \varphi_{0} J_{0}^{2}\left(4 \hat{\phi}_{\mathrm{F}}\right)}$. 
It is now possible to solve equations $((6 a)$ and $(6 b))$ with respect to the ellipticity angle and the operating point. We obtain

$$
\begin{gathered}
X_{\phi}=\frac{\chi \sqrt{1-J_{0}\left(8 \hat{\phi}_{\mathrm{F}}\right)}}{\sqrt{8 I_{\mathrm{ac}}^{2} J_{0}^{2}\left(4 \hat{\phi}_{\mathrm{F}}\right)+2 \chi^{2}\left[J_{0}^{2}\left(4 \hat{\phi}_{\mathrm{F}}\right)-J_{0}\left(8 \hat{\phi}_{\mathrm{F}}\right)\right]}} \\
X_{\varepsilon}=\frac{\chi}{X_{\phi} I_{0} J_{0}\left(4 \hat{\phi}_{\mathrm{F}}\right)}
\end{gathered}
$$

where $X_{\phi}=\cos 2 \phi, X_{\varepsilon}=\cos 2 \varepsilon$ and $\chi=2 I_{\mathrm{dc}}-I_{0}$.

$I_{0}$ represents the total intensity of light just before the polarizer and is measured by a second photodetector (PD2). The gains of the two photodetectors are known with sufficient precision, but electronics may introduce different gains in processing physical data. For a correct interpretation of the information on $I_{0}$ we need to calculate a correction factor.

Using the Jacobi-Anger expansion from equation (5) it is possible to calculate analytically the amplitude of the second harmonic, which is measured with a lock-in amplifier:

$$
I_{2 f}^{\text {meas }}=I_{0} \cos 2 \varepsilon \cos 2 \varphi_{0} J_{2}\left(4 \hat{\phi}_{\mathrm{F}}\right) .
$$

Combining (8) with $(6 a)$ it is also possible to deduce an expression where the second harmonic is theoretically calculated from only measured quantities:

$$
I_{2 f}^{\text {th }}=\chi \frac{J_{2}\left(4 \hat{\phi}_{\mathrm{F}}\right)}{J_{0}\left(4 \hat{\phi}_{\mathrm{F}}\right)} .
$$

Fitting by least squares the measured second harmonic (8) with the theoretically calculated value (9) will give the correction factor for the intensity $I_{0}$.

\section{AC current measurement}

The signal processing given by equation (1) is poorly efficient when far from the ideal settings. Even if a good approximation using a third-order polynomial is possible, it does not take into account any variation of the operating point or the ellipticity (due for example to temperature changes).

A different way to approach the problem consists in inverting equation (5) with respect to the electrical current, as follows:

$$
I_{\mathrm{el}}^{\mathrm{ac}}=\frac{1}{4 N V}\left[\arccos \left(\frac{2 I_{\mathrm{out}}-I_{0}}{X_{\varepsilon} I_{0}}\right)-\arccos X_{\phi}\right]
$$

where $I_{\mathrm{out}}=I_{\mathrm{dc}}-I_{\mathrm{ac}} \sqrt{2}$ is the output intensity.

The operating point $X_{\phi}$ and the ellipticity angle $X_{\varepsilon}$ are previously calculated using equations (7) by mean of an ac calibration, after having properly calculated the correction coefficient for $I_{0}$.

Figure 5 displays a measurement of the optical-electrical transfer function of the sensor obtained with an imperfect adjustment of the polarization controllers: the calculated parameters $\phi_{0}=44.72^{\circ}$ and $\varepsilon=18.23^{\circ}$ demonstrate that, even if the setting could be improved, the sensor exhibits an excellent linearity over more than three decades. Figure 6 shows the difference between the optically measured current and the reference current: the error points stay within $2 \mathrm{~A}$ and -2 A (dotted lines), meaning that the accuracy is better than $0.2 \%$ at the maximum value of the scale.

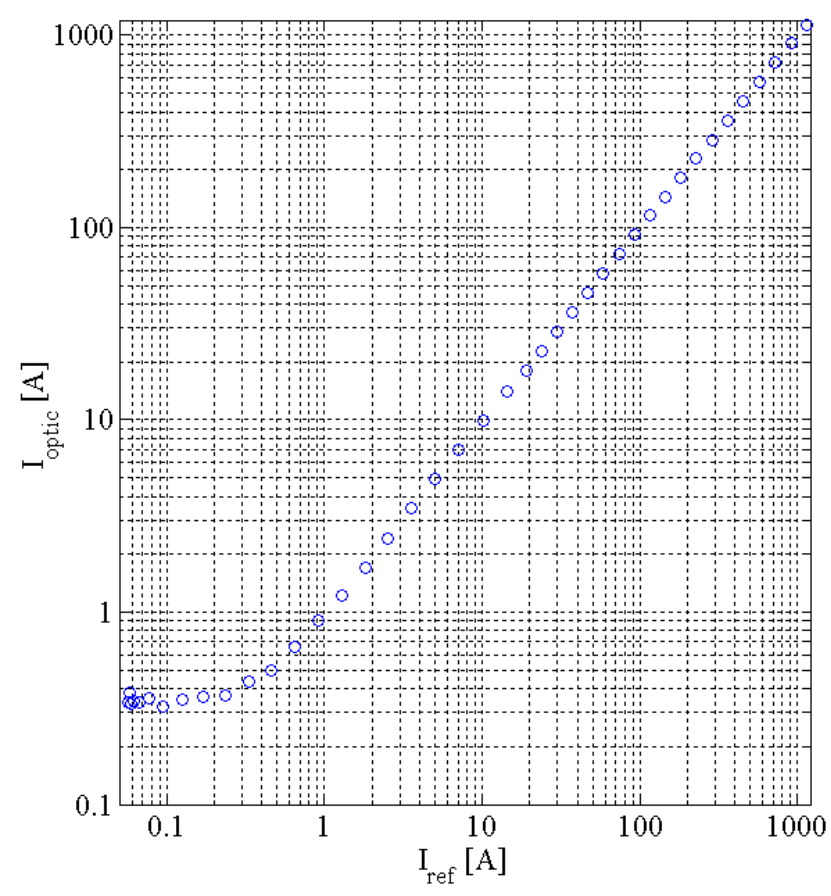

Figure 5. Optical-electrical ac transfer function obtained with an imperfect adjustment of the polarization controllers $\left(\phi_{0}=44.72^{\circ}\right.$ and $\varepsilon=18.23^{\circ}$ ): the sensor exhibits an excellent linearity over more than three decades. The reference current is obtained by measuring the voltage on a serial resistor.

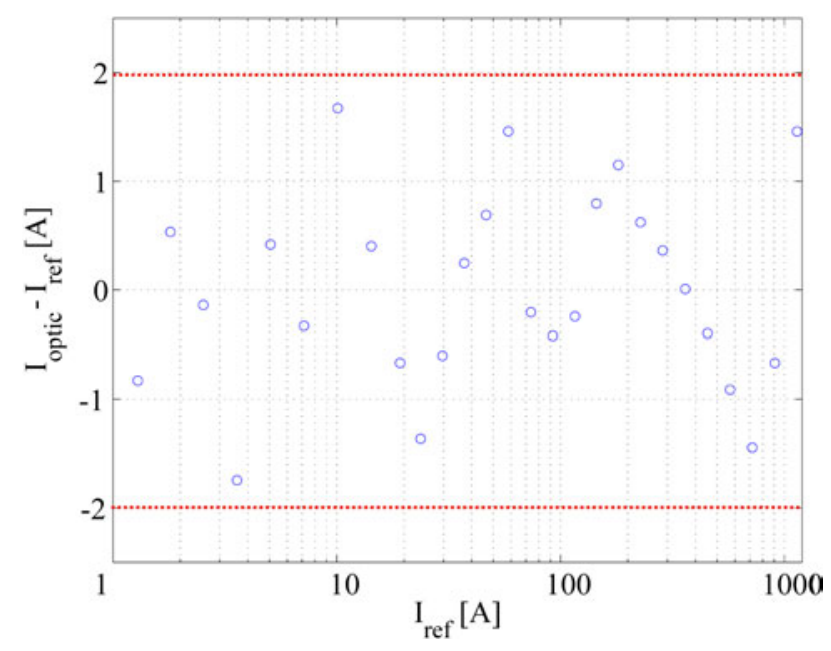

Figure 6. Difference between the optically measured current and the reference current. The error points stay within $2 \mathrm{~A}$ and $-2 \mathrm{~A}$, meaning that the accuracy is better than $0.2 \%$ at the maximum value of the scale.

\section{Extension to de currents}

The approach described by equation (10) offers the advantage of being easily applied to the measure of dc currents, with no significant modifications. The ac components being absent from the output intensity signal, we have simply $I_{\text {out }}=I_{\mathrm{dc}}$ and consequently

$$
I_{\mathrm{el}}^{\mathrm{dc}}=\frac{1}{4 N V}\left[\arccos \left(\frac{2 I_{\mathrm{dc}}-I_{0}}{X_{\varepsilon} I_{0}}\right)-\arccos X_{\phi}\right]
$$

where the operating point $X_{\phi}$, the ellipticity angle $X_{\varepsilon}$ and the correction coefficient for $I_{0}$ are the same as those used for the 


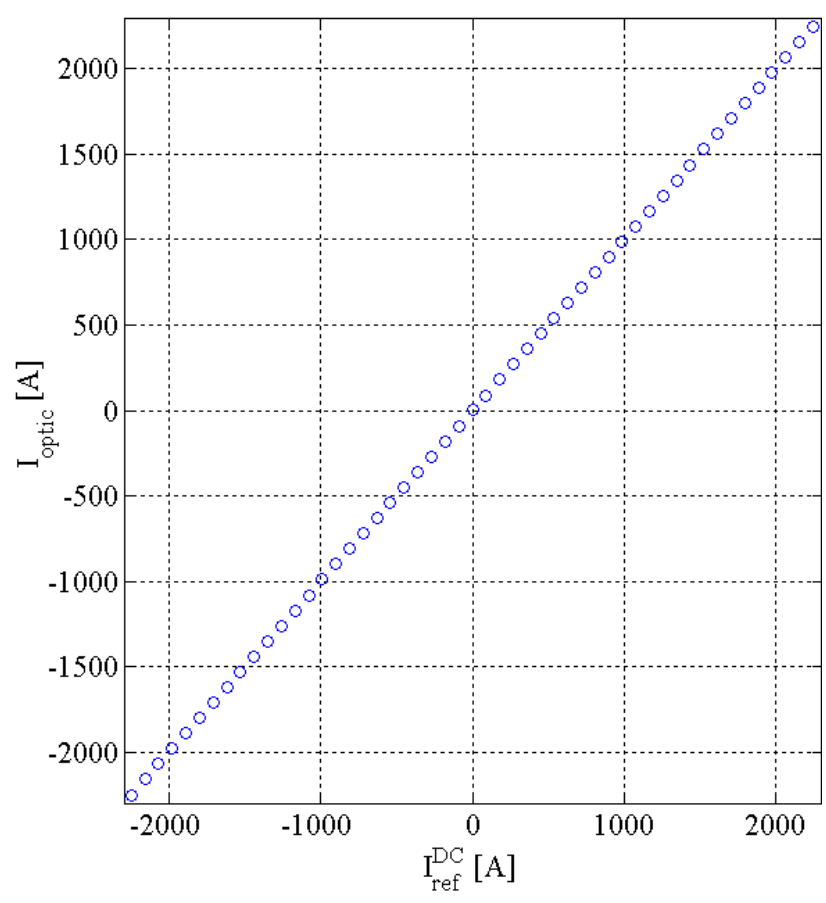

Figure 7. Optical-electrical dc transfer function obtained with an imperfect adjustment of the polarization controllers $\left(\phi_{0}=44.72^{\circ}\right.$ and $\varepsilon=18.23^{\circ}$ ). A scale factor correction of 0.92 is applied. The reference current is obtained by measuring the voltage on a serial resistor.

measurement of ac currents. The optical-electrical dc transfer function shown in figure 7 is intrinsically linear: only a slight scale factor correction has been included which takes into account any imprecision on knowledge of the Verdet constant.

\section{Modelling of thermal effects}

The sensor modelling presented previously allowed us to completely characterize the system and to obtain very good measurement accuracy through a precise knowledge of the operating point and the output polarization state.

Ideally, the calibration parameters $\left(\varphi_{0}\right.$ and $\left.\varepsilon\right)$ are calculated once and for all and should not change during time. However, the presence of elements sensitive to temperature, for instance, makes the system subject to variations in the environmental conditions.

After having studied the thermal effects on the different elements constituting the sensor, we are able to localize the most sensitive to temperature, but we cannot model the global behaviour in an analytical way because of the correlation existing between the physical variables. By observing the evolution of the calibration parameters under the influence of a random environmental temperature change (see figure 8), we noted that there is a tight correlation between $\varphi_{0}$ and $\varepsilon$ on one side and the dc and ac components of the output intensity on the other side, this even over a temperature variation of only $2{ }^{\circ} \mathrm{C}$. A temperature variation can thus be taken into account in an empirical way to compensate its effect on the measurement of the electric current. The relations will have
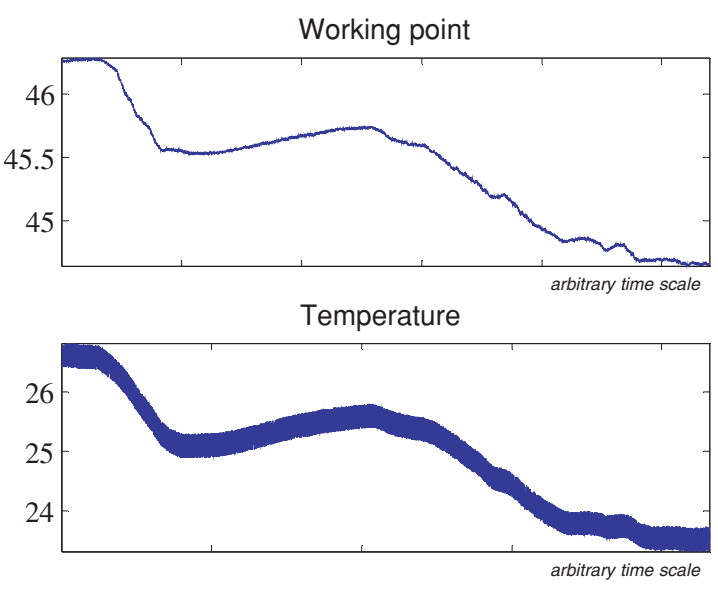

Figure 8. Evolution of the operating point according to a random environmental temperature variation.

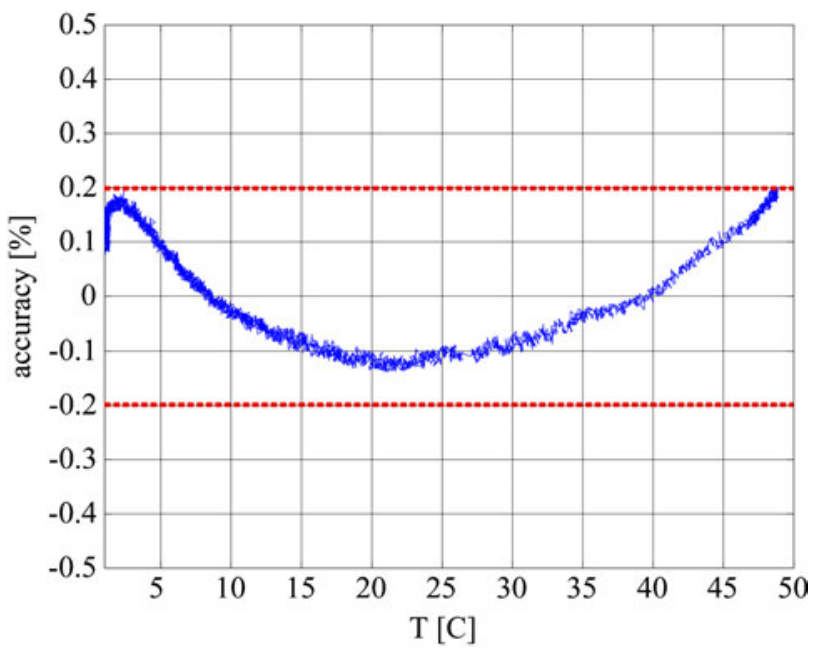

Figure 9. Measurement accuracy with respect to temperature variation. The reference current is set to a constant value $(1.22 \mathrm{kA})$ and the temperature is changed over $50{ }^{\circ} \mathrm{C}$. The $\pm 0.2 \%$ accuracy is represented by dotted lines.

the following form:

$$
\begin{gathered}
\varphi_{0}(T)=\varphi_{0}\left(T_{0}\right) \frac{f_{\varphi}\left(I_{\mathrm{dc}}\left(T_{0}\right), I_{0}\left(T_{0}\right), I_{\mathrm{ac}}\left(T_{0}\right)\right)}{g_{\varphi}\left(I_{\mathrm{dc}}(T), I_{0}(T), I_{\mathrm{ac}}(T)\right)} \\
X_{\varepsilon}(T)=X_{\varepsilon}\left(T_{0}\right) \frac{f_{\varepsilon}\left(I_{\mathrm{dc}}\left(T_{0}\right), I_{0}\left(T_{0}\right), I_{\mathrm{ac}}\left(T_{0}\right)\right)}{g_{\varepsilon}\left(I_{\mathrm{dc}}(T), I_{0}(T), I_{\mathrm{ac}}(T)\right)} .
\end{gathered}
$$

Equations (12) clearly show that the values updated for $\varphi_{0}$ and $\varepsilon$ are calculated from the values measured during the calibration for a given temperature, as a function of only $I_{\mathrm{dc}}$, $I_{\mathrm{ac}}$ and $I_{0}$. The independence of the reference current $I_{\mathrm{ref}}$ allows real-time compensation for any range of measurement. A very good estimate for the calculation of $\varphi_{0}$ and $\varepsilon$ is given by the following formulae:

$$
\begin{gathered}
\varphi_{0}(T)=\varphi_{0}\left(T_{0}\right) \sqrt{\frac{I_{\mathrm{dc}}\left(T_{0}\right)}{I_{\mathrm{dc}}(T)} \sqrt{\frac{I_{0}\left(T_{0}\right)}{I_{0}(T)}}} \\
X_{\mathcal{\varepsilon}}(T)=X_{\varepsilon}\left(T_{0}\right) \frac{I_{\mathrm{ac}}\left(T_{0}\right)}{I_{\mathrm{ac}}(T)} \frac{I_{0}\left(T_{0}\right)}{I_{0}(T)} .
\end{gathered}
$$

This makes it possible to always keep measurement accuracy within $0.2 \%$ as shown in figure 9 , where the 
temperature is changed over $50{ }^{\circ} \mathrm{C}$ and the reference current is set to a constant value (1.22 $\mathrm{kA}$ in our special case). A little more precise relationship could be extracted from formulae (12), but one should keep in mind that there remains a threshold of minimal error imposed by the rate of mechanical twisting of the twisted fibre. With the relations (13) a good trade-off between precision and calculation complexity is obtained.

\section{Conclusions}

In this paper we presented a polarimetric current sensor in an original new all-fibre configuration, offering the key advantage of compensating any stray non-random birefringence induced by additional elements such as connecting fibres and connectors. This results in a very flexible configuration with a sensing fibre that may be wrapped around the conductor and then be connected to the instrument in the field. It makes this kind of sensor definitely portable, requiring no interruption of the electrical conductor.

The configuration is easy to implement, at the expense of a more complex adjustment and signal processing. The all-fibre configuration makes the instrument very stable and immune to environmental issues and is therefore particularly well matched to the end-user requirements (flexibility in the installation procedure and immunity to humidity, dust and temperature).

The measurement of ac currents is presented with an excellent linearity over more than three decades and a good accuracy (better than $0.2 \%$ ) at the maximum value of the scale. Thanks to a further developed signal processing, the measure of dc currents is also demonstrated with the same accuracy. To take into account the variation of the calibration parameters with temperature, an empirical model is also proposed granting a precision within $0.2 \%$ over a wide temperature range.

\section{References}

[1] Briffod F, Alasia D, Thévenaz L, Cuénoud G and Robert P-A 2002 Extreme current measurements using a fibre optics current sensor Technical Digest of the 15th Optical Fibre Sensors Conf. OFS'2002 (Portland, OR, USA) IEEE Catalogue number 02EX533, Post-deadline paper (PD3)

[2] Rose A H, Deeter M N and Day G W 1993 Submicroampere-per-root-hertz current sensor based on the Faraday effect in Ga:YIG Opt. Lett. 18 1471-3

[3] Nicati P-A and Robert P-A 1988 Stabilized current sensor using Sagnac interferometer J. Phys. E: Sci. Instrum. 21 791-6

[4] Patterson R L, Rose A H, Tang D and Day G W 1990 A fibre-optic current sensor for aerospace applications Presented at IEEE AES Systems Magazine (December)

[5] Nicati P-A and Robert P-A 1992 Stabilized Sagnac optical fibre current sensor using one phase and two amplitude modulations Proc. 8th Optical Fibre Sensors Conf. (Monterey, CA, January) pp 402-5

[6] Tang D, Rose A H, Day G W and Etzel S 1991 Annealing of linear birefringence in single-mode fibre coils: application to optical fibre current sensors J. Lightwave Technol. 9 $1031-7$

[7] Ulrich R and Simon A 1979 Polarization optics of twisted single-mode fibres Appl. Opt. 18 2241-51

[8] Briffod F, Thévenaz L, Nicati P-A, Küng A and Robert P-A 2000 Polarimetric current sensor using an in-line Faraday rotator IEICE Trans. Electron. 83 331-4

[9] Briffod F 2003 Structures innovantes pour capteurs optiques de courants électriques $P h D$ Thesis Swiss Federal Institut of Technology, Lausanne

[10] Pistoni N and Martinelli M 1993 Vibration-insensitive fibre-optic current sensor Opt. Lett. 18 314-6 